Pacific Journal of Mathematics

CONCERNING MEASURES ON BOOLEAN ALGEBRAS 


\section{CONCERNING MEASURES ON BOOLEAN ALGEBRAS}

\section{HAIM GAIFMAN}

Introduction. The main aim of this work is to show that certain conditions are not sufficient for a Boolean algebra to have a strictly positive measure, where by a measure we mean a finitely additive measure which assigns 1 to the maximal element, and a strictly positive measure is one which assigns values $>0$ to every nonzero element. The results remain the same if the maximal element is allowed to have any finite measure $>0$, or an infinite measure provided that some nonzero element has a finite measure. We construct a Boolean algebra whose set of elements is a denumerable union of the form $\bigcup_{n=1}^{\infty} B_{n}$ where in $B_{n}$ there are at most $n$ pairwise disjoint elements, but which has no strictly positive measure. This also implies that the countable chain condition, i.e. the condition that there are only countably many pairwise disjoint elements in the Boolean algebra, is not sufficient for the existence of a strictly positive measure. (The existence of sets $B_{1}, \cdots, B_{n}, \cdots$ having the above mentioned properties is implied by the existence of a strictly positive measure and implies the countable chain condition.)

The problem of the sufficiency of the countable chain condition was raised by Tarski in the thirties [9 p. 58]; the second stronger condition was formulated by Horn and Tarski in 1948 [3, pp. $481 \mathrm{ff}$ ], where the problem of its sufficiency as well as the sufficiency of other conditions each of which is implied by it and implies the countable chain condition, is posed. A condition of a more complicated character which is both necessary and sufficient for the existence of a strictly positive measure was given by Kelley in 1959 [4]. Note that a characterization of the Boolean algebras which admit a strictly positive measure amounts to a characterization of those ideals which, for some measure, are the ideals of all elements of measure 0 . This is so since every measure induces in a natural way a strictly positive measure on the quotient algebra of the original algebra and the ideal of all elements of measure 0 . The results and proofs can be reformulated in an obvious way so as to deal with arbitrary measures and the ideals of all elements of measure 0 .

Whether the countable chain condition implies the second stronger condition, mentioned above, is unknown and seems unlikely as this would imply Souslin's conjecture, cf. [3 pp. $487 \mathrm{ff}$.$] .$

Received May 15, 1963. The results of this work constitute part of the author's dissertation done under the supervision of Prof. Tarski. The author wishes to thank Prof. Horn for his help in checking the proof of the main result. 
In $\S 1$ there are some preliminary definitions and lemmas, and $\S 2$ includes the proof of the main result and some corollaries. In $\S 3$ the analogous problems for countably additive Boolean algebras and countably additive measures are pointed out. In $\S 4$ several connections between certain problems concerning Boolean algebras and Souslin's conjecture are discussed. The theorem whose proof is outlined there shows that Souslin's conjecture is equivalent to some relativity simple statements concerning countably additive Boolean algebras.

Notation. A Boolean algebra is conceived here as an algebra of the form $\langle B ; \vee, \wedge,-\rangle$ where $\vee$ and $\wedge$ are the join and meet operations, respectively, and - is the complementation operation. All the well known properties of these operations are assumed. If $\mathfrak{B}=$ $\langle B ; \vee, \wedge,-\rangle$ then $|\mathfrak{B}|=B$, i.e. the set of all elements of the Boolean algebra. The partial ordering on $|\mathfrak{B}|$ is denoted by " $\geqq$ ", and " 1 " and " 0 " denote the maximal and minimal elements respectively. If $\left\{a_{i}\right\}_{i \in I} \subseteq$ $|\mathfrak{B}|$ and the least upper bound of $\left\{a_{i}\right\}_{i \in I}$ exists, then it is referred to as the join of $\left\{a_{i}\right\}_{i \in I}$ and denoted by " $\bigvee_{i \in I} a_{i}$ ". Similarly " $\bigwedge_{i \in I} a_{i}$ " denotes the greatest lower bound of $\left\{a_{i}\right\}_{i \in I}$, when it exists, and is referred to as the meet of $\left\{a_{i}\right\}_{i \in I}$. A Boolean algebra is complete if the joins and meets of all sets of its elements exist. A set $B$ of elements of $\mathfrak{B}$ is dense in $\mathfrak{B}$ if for every nonzero $b \in|\mathfrak{B}|$ there is a nonzero $b^{\prime} \in B$ such that $b \geqq b^{\prime}$. Every Boolean algebra $\mathfrak{B}$ can be embedded as a subalgebra in a complete Boolean algebra $\mathfrak{B}^{\prime}$ so that $|\mathfrak{B}|$ is dense in $\mathfrak{B}^{\prime}$. The Boolean algebra $\mathfrak{B}^{\prime}$ (which is unique up to an isomorphism) is the completion by cuts of $\mathfrak{B}$. If $b \in|\mathfrak{B}|$ then $\left.\mathfrak{B}\right|_{b}$ is the Boolean algebra formed by the principal ideal generated by $b$, i.e. $\left.\mathfrak{B}\right|_{b}=\left\langle B^{\prime}, \vee^{\prime}, \wedge^{\prime},{ }^{-}\right\rangle$, where $B^{\prime}=\{c \mid c \leqq b\}, \vee^{\prime}$ and $\wedge^{\prime}$ are $\vee$ and $\wedge$, respectively, restricted to $B^{\prime}$, and $\bar{c}^{\prime}=\bar{c} \wedge b$ for all $c \in B^{\prime}$.

We will always exclude the trivial Boolean algebra of one element, that is, we assume that $1 \neq 0$.

\section{Section 1.}

Definition. A measure $m$ on a Boolean algebra $\mathfrak{B}$ is a function from $|\mathfrak{B}|$ into $[0,1]$ such that $m(1)=1$ and $m(a \vee b)=m(a)+m(b)$ whenever $a \wedge b=0$. A measure $m$ is strictly positive if $m(a) \neq 0$ whenever $a \neq 0$.

We will assume all the well known properties of measures such as $m(0)=0, m\left(a_{1} \vee \cdots \vee a_{n}\right)=\sum_{i=1}^{n} m\left(a_{i}\right)$ if $a_{i} \wedge a_{j}=0$ whenever $i \neq j$, etc.

A set $B$ of elements of a Boolean algebra is a set of pairwise disjoint elements or, simply, disjoint elements if for all $a, b$ in $B$ either $a \wedge b=0$ or $a=b$. B has at most $n$ disjoint elements if every 
subset of $B$ of disjoint elements is of power $\leqq n$.

A Boolean algebra satisfies the C.C.C. (countable chain condition) if every set of disjoint elements of this algebra is countable (i.e. finite, or infinite and denumerable).

If $m$ is a strictly positive measure on $\mathfrak{B}$ and if $B_{1}=\{\mathbf{0}\}$ and $B_{n}=$ $\{b \mid m(b) \geqq(1 / n)\}$ for $n>1$, then we get $|\mathfrak{B}|=\mathbf{U}_{n=1} B_{n}$ and, as is easily seen, $B_{n}$ has at most $n$ disjoint elements. Consequently a necessary condition for a Boolean algebra to have a strictly positive measure is: (*) There are sets $B_{1}, B_{2}, \cdots, B_{n}, \cdots, 0<n<\infty$, such that for every $n$ the set $B_{n}$ has at most $n$ disjoint elements, and $\bigcup_{n=1}^{\infty} B_{n}$ is the set of all the elements of the Boolean algebra.

$(*)$ is easily seen to imply the C.C.C. since if $B$ is a set of pairwise disjoint elements and $B_{1}, B_{2} \cdots, B_{n}, \cdots$ are as in $(*)$, then $B=$ $\mathrm{U}_{n=1}^{\infty}\left(B \cap B_{n}\right)$ and $B \cap B_{n}$ is at most of power $n$. Hence $B$ is countable. Thus we get

LEMmA 1.1. There exists a strictly positive measure $\Rightarrow(*) \Rightarrow$ C.C.C.

Our main result is to show that $(*) \nRightarrow$ there exists a strictly positive measure. To show that C.C.C. $\Rightarrow(*)$ is an open problem which will be discussed in $\S 4$.

LEMMA 1.2. If there are sets $B_{1}, \cdots, B_{i}, \cdots, 0<i<\infty$ such that $|\mathfrak{B}|=\bigcup_{i=1}^{\infty} B_{i}$ and such that in every $B_{i}$ there are at most $n_{i}$ disjoint elements where $n_{1}, \cdots, n_{i}, \cdots$ is a sequence of natural numbers, then $\mathfrak{B}$ satisfies $(*)$.

Proof. Given any natural number $k$ if there is no number $j$ such that $k \geqq \sum_{i=1}^{j} n_{i}$ put $B_{k}^{\prime}=\phi$; otherwise let $j(k)$ be the largest $j$ for which $k \geqq \sum_{i=1}^{j} n_{i}$ and put $B_{k}^{\prime}=\bigcup_{i \leqq j(k)} B_{i}$. Obviously $B_{l k}^{\prime}$ has at most $k$ disjoint elements and $|\mathfrak{B}|=\bigcup_{n=1}^{\infty} B_{n}^{\prime}$.

Definition. (i) If $\left\langle b_{1}, \cdots, b_{n}\right\rangle$ is an $n$-tuple of elements of a Boolean algebra. Let $N\left(\left\langle b_{1}, \cdots, b_{n}\right\rangle\right)$ be the maximal number $k$ for which there are $b_{i_{1}}, \cdots, b_{i_{k}}$ such that $1 \leqq i_{1}<i_{2}<\cdots<i_{k} \leqq n$ and $b_{i_{1}} \wedge b_{i_{2}} \wedge \cdots \wedge b_{i_{k}} \neq 0$.

(ii) If $B \subseteq|\mathfrak{B}|$ then the intersection number of $B$, Int $(B)$, is defined as $\inf \left\{(1 / n) N\left(\left\langle b_{1}, \cdots, b_{n}\right\rangle\right) \mid b_{1}, \cdots, b_{n} \in B\right\}$, where the infinum is taken over all finite sequences of elements of $B$.

This definition as well as the following two lemmas are due to Kelley [4]. The lemmas constitute the easier part of his result and we give here their simple proofs.

LEMMA 1.3. If $m$ is a measure on $\mathfrak{B}$ and $B \subseteq|\mathfrak{B}|$ then $\operatorname{Int}(B) \geqq$ 
$\operatorname{Inf}\{m(b) \mid b \in B\}$.

Proof. Assume $\operatorname{Inf}\{m(b) \mid b \in B\}=\delta$. Let $b_{1}, \cdots, b_{n} \in B$. For every subset $K$ of $\{1, \cdots, n\}$ put $a_{K}=\Lambda_{k \in K} b_{k} \wedge \Lambda_{k \in \bar{K}} \bar{b}_{k}$, where $\bar{K}$ is the complement of $K$ relative to $\{1, \cdots, n\}$. Obviously $a_{K} \wedge a_{K^{\prime}}=0$ if $K \neq K^{\prime}$ and $b_{k}=\mathbf{V}_{k \in K} a_{K}$. Hence $m\left(b_{k}\right)=\sum_{k \in K} m\left(a_{K}\right)$ and $\sum_{k=1}^{n} m\left(b_{k}\right)=$ $\sum_{k=1}^{n} \sum_{k \in K} m\left(a_{K}\right)=\sum_{K} r(K) \cdot m\left(a_{K}\right)$, where $r(K)=0$ if $a_{K}=0$ and $r(K)=$ the cardinality of $K$ if $a_{K} \neq 0$. Put $r=\max \{r(K) \mid K \cong\{1, \cdots, n\}$. Then $r \leqq N\left(\left\langle b_{1}, \cdots, b_{n}\right\rangle\right)$ and $n \delta \leqq \sum_{k=1}^{n} m\left(b_{k}\right)=\sum_{K} r(K) \cdot m\left(a_{K}\right) \leqq$ $r \cdot \sum_{K} m\left(a_{K}\right) \leqq r \quad\left(\right.$ since $\left.\quad \sum_{K} m\left(a_{K}\right)=m\left(\mathbf{V}_{K} a_{K}\right) \leqq 1\right)$. Consequently $(1 / n) N\left(\left\langle b_{1}, \cdots, b_{n}\right\rangle\right) \geqq \delta$. Since this is true for all $b_{1}, \cdots, b_{n}$ in $B$ we get $\operatorname{Int}(B) \geqq \delta$.

LEMMA 1.4. If $\mathfrak{B}$ has a strictly positive measure then there are sets $B_{1}, \cdots, B_{n}, \cdots, 0<n<\infty$ such that $|\mathfrak{B}|=\{\mathbf{0}\} \cup \bigcup_{n=1}^{\infty} B_{n}$ and. $\operatorname{Int}\left(B_{n}\right)>0$ for all $n$.

Proof. Put $B_{n}=\{b \mid m(b) \geqq(1 / n)\}$, where $m$ is the strictly positive measure, and use Lemma 1.3.

We will construct a Boolean algebra which satisfies $(*)$, but which has no decomposition of the form $\{0\} \cup \bigcup_{n=1}^{\infty} B_{n}$ where $\operatorname{Int}\left(B_{n}\right)>0$. This Boolean algebra will have no strictly positive measure. Kelley's result is that the existence of a decomposition of that form is also sufficient for the existence of a strictly positive measure.

\section{Section 2.}

Definition. A set $B$ of elements of a Boolean algebra is a set. of independent elements or, simply, independent if whenever $\left\{b_{1}, \cdots, b_{k}\right.$, $\left.b_{k+1}, \cdots, b_{n}\right\} \subseteq B$ and $b_{i} \neq b_{j}$ for $i \neq j$ we have $\bigwedge_{i=1}^{k} b_{i} \wedge \bigwedge_{i=k+1}^{n} \bar{b}_{i} \neq 0$.

The following are well known properties of independent sets. Let. $B$ be an independent set, then:

( $\alpha)$ If $\bigwedge_{i=1}^{k} b_{i} \wedge \bigwedge_{i=1}^{n} \bar{b}_{i}^{\prime} \leqq \bigwedge_{i=1}^{m} c_{i}$, where $b_{1}, \cdots, b_{k}, b_{1}^{\prime}, \cdots, b_{n}^{\prime}$ and $c_{1}, \cdots, c_{m}$. are all members of $B$, then either for some $i$ and $j b_{i}=b_{j}^{\prime}$ (in which. case the meet on the left side is 0 ), or $\left\{b_{1}, \cdots, b_{k}\right\} \supseteqq\left\{c_{1}, \cdots, c_{m}\right\}$.

$(\beta)$ If $a_{1}, \cdots, a_{m}$ are finite meets of members of $B$ and $b$ is a finite: meet of members of $B$ and complements of members of $B$, then $b \leqq$ $\bigvee_{i=1}^{m} a_{j}$ only if $b \leqq a_{i}$ for some $i, 1 \leqq i \leqq m$.

$(\gamma)$ If $B_{n}$ is the set of all elements of the form $\bigwedge_{i=1}^{k} b_{i} \wedge \bigwedge_{i=k+1}^{m} \bar{b}_{i}$ : where $\left\{b_{1}, \cdots, b_{m}\right\} \subseteq B$ and $m \leqq n$, then in $B_{n}$ there are at most $2^{n}$. nonzero disjoint elements.

In the theorem that follows we use the free Boolean algebra on continuum many generators. This is the Boolean algebra generated by continuum many generators in which the set of the generators is 
independent. The existence of a free Boolean algebra on a given number of generators is a well known consequence of a general result concerning free algebras, cf. Birkhoff [2], and [1, p. viii ff.]. Moreover, in the case of continuum many generators this Boolean algebra can be actually given as an algebra of subsets of a denumerable set (the Boolean operations being the usual set operations). It is known that there is an independent family of continuum many subsets of a denumerable set (cf. [8 p. 6] where other references can be found) and one has to take the Boolean algebra generated by this family. For instance, take the denumerable set to be the set of all sets of the form $\left\{T_{1}, \cdots, T_{n}\right\}$ where $n$ is any natural number and $T_{1}, \cdots, T_{n}$ are open intervals in $(0,1)$ with rational end points. For every $x \in(0,1)$ let $S_{x}$ be the subset of all elements $\left\{T_{1}, \cdots, T_{n}\right\}$ such that $x \in T_{1} \cup \cdots \cup T_{n}$. Then $\left\{S_{x} \mid x \in(0,1)\right\}$ is an independent family of continuum many elements.

THEOREM 2.1. There exists a Boolean algebra which satisfies (*) (i.e., the set of all its elements can be represented as $\bigcup_{i=1}^{\infty} B_{n}$, where in $B_{n}$ there are at most $n$ disjoint elements) and which has no strictly positive measure.

The proof is divided into 3 parts. The first is a construction of a certain ideal $\mathfrak{\Im}$ in the free Boolean algebra on continuum many generators, $\mathfrak{B}$. The second part is a proof that $\mathfrak{B} / \mathfrak{\Im}$ satisfies $(*)$ and in the third part we show that $\mathfrak{B} / \mathfrak{\Im}$ has no strictly positive measure.

(I) Construction. Let $\mathfrak{B}$ be the free Boolean algebra on continuum many generators. Let the set of free generators be $\left\{b_{x} \mid x \in(0,1)\right\}$ where $b_{x} \neq b_{y}$ if $x \neq y$. Enumerate all nonempty open intervals of $(0,1)$ with rational end points; it is convenient to start the enumeration from 2 ; let it be $T_{2}, T_{3}, \cdots, T_{n}, \cdots, 2 \leqq n<\infty$. For every $i \geqq 2$ let $T_{i, 1}, \cdots, T_{i, i^{2}}$ be a sequence of $i^{2}$ pairwise disjoint nonempty open subintervals of $T_{i}$ with rational end points. (The number $i^{2}$ has no special significance and is chosen for convenience. The same proof works if we put $n_{i}$ instead of $i^{2}$ provided that $n_{i} \geqq i$ for $i \geqq 2$ and that $i / n_{i} \rightarrow 0$ as $i \rightarrow \infty$.)

For every $i \geqq 2$ let $H_{i}$ be the set of all elements of $\mathfrak{B}$ of the form $b_{x_{1}} \wedge \cdots \wedge b_{x_{i}}$ where $x_{1}, \cdots, x_{i}$ belong to $i$ different intervals of the form $T_{i, j}, 1 \leqq j \leqq i^{2}$. Put $H=\bigcup_{i=2}^{\infty} H_{i}$ and let $\mathfrak{\Im}$ be the ideal generated by $H$.

During the proof $x, x_{1}, \cdots, y, y_{1}, \cdots, z, z_{1}, \cdots$ range over points

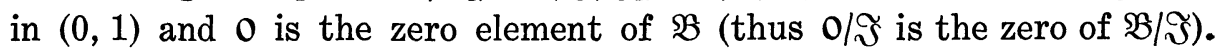

(II) $\mathfrak{B} / \Im$ satisfies $(*)$ 
Proof. A member $b$ of $\mathfrak{B}$ is in $\mathfrak{\Im}$ if and only if $b \leqq a_{1} \vee \cdots \vee a_{m}$ for some $a_{1}, \cdots, a_{m}$ in $H$. Assume that $b=b_{x_{1}} \wedge \cdots \wedge b_{x_{k}} \wedge \bar{b}_{y_{1}} \wedge \cdots \wedge \bar{b}_{y_{n}}$ ( $n$ may be 0 ). Since every member of $H$ is a meet of free generators it follows from $(\beta)$ that $b \in \mathfrak{\Im}$ if and only if for some $a$ in $H, b \leqq a$. Let $a=b_{z_{1}} \wedge \cdots \wedge b_{z_{i}}$, where $z_{1}, \cdots, z_{i}$ belong to $i$ different intervals of the form $T_{i, j}$. From $(\alpha)$ it follows that $b \leqq a$ if and only if either $b=0$ or $\left\{x_{1}, \cdots, x_{k}\right\} \supseteqq\left\{z_{1}, \cdots, z_{i}\right\}$. This proves the following:

(i) If $b_{x_{1}} \wedge \cdots \wedge b_{x_{k}} \wedge \bar{b}_{y_{1}} \wedge \cdots \wedge \bar{b}_{y_{n}} \in \Im$, then either this element is 0 or there is an $i$ such that $k \geqq i \geqq 2$ and among $x_{1}, \cdots, x_{k}$ there are $i$ points which belong to $i$ different intervals of the form $T_{i, j}$, $\left(1 \leqq j \leqq i^{2}\right)$. Obviously, the converse holds as well.

(ii) Let $b^{\prime}$ and $b^{\prime \prime}$ be meets of complements of the free generators and let $b_{1}=b_{x_{1}} \wedge \cdots \wedge b_{x_{m}} \wedge b^{\prime}$ and $b_{2}=b_{y_{1}} \wedge \cdots \wedge b_{y_{n}} \wedge b^{\prime \prime}\left(b^{\prime}\right.$ or $b^{\prime \prime}$ may be absent from $b_{1}$ or $b_{2}$ ). Assume that $b_{1} \notin \mathfrak{F}$ and that for every $i$ and $j$ such that $2 \leqq i \leqq m+n$ and $1 \leqq j \leqq i^{2}$ if there is a $k$ such that $y_{k} \in T_{i, j}, \quad(1 \leqq k \leqq n)$ then there is a $k^{\prime}$ such that $x_{k^{\prime}} \in T_{i, j}$, $\left(1 \leqq k^{\prime} \leqq m\right)$. Under these assumptions $b_{1} \wedge b_{2} \in \mathfrak{F}$ only if $b_{1} \wedge b_{2}=0$.

(ii) is implied by (i): if $b_{1} \wedge b_{2} \in \mathfrak{\Im}$ and $b_{1} \wedge b_{2} \neq 0$ then there is an $i, 2 \leqq i \leqq m+n$, such that among $x_{1}, \cdots, x_{m}, y_{1}, \cdots, y_{n}$ there are $i$ points which belong to $i$ different intervals of the form $T_{i, j}$. Since every $y_{k}$ which belongs to some $T_{i, j}$ can be replaced by $x_{k^{\prime}}$ which belongs to the same $T_{i, j}$, it will follow that $b_{1} \in \mathfrak{S}$, contradicting our assumption.

For $n \geqq 2$ let $K_{n}$ be the set of all ordered pairs $\langle i, j\rangle$ where $2 \leqq i \leqq n$ and $1 \leqq j \leqq i^{2}$. If $K \leqq K_{n}$ let $C(K, n)$ be the set of all members of $\mathfrak{B}$ which are not in $\Im$ and are of the form: $b_{x_{1}} \wedge \cdots \wedge b_{x_{m}} \wedge$ $\bar{b}_{y_{1}} \wedge \cdots \wedge \bar{b}_{y_{m^{\prime}}}$, (including the cases $m=0$ and $m^{\prime}=0$ ) where $m+m^{\prime} \leqq n / 2$ and, for all $i$ and $j$ such that $\langle i, j\rangle \in K_{n},\langle i, j\rangle \in K$ if and only if $x_{r} \in T_{i, j}$ for some $r(1 \leqq r \leqq m)$.

From (ii) it follows that whenever $b_{1}$ and $b_{2}$ are members of $C(K, n)$ such that $b_{1} \wedge b_{2} \in \mathfrak{\Im}$, then $b_{1} \wedge b_{2}=0$. Hence, by $(\gamma)$, every subset of $C(K, n)$, in which the meet of every two different elements is in $\Im$, is of power $\leqq 2^{n / 2}$.

Let $C_{n}$ be the set of all members of $\mathfrak{B}$ which are not in $\mathfrak{\Im}$ and which are of the form $b_{x_{1}} \wedge \cdots \wedge b_{x_{k}} \wedge \bar{b}_{x_{k+1}} \wedge \cdots \wedge \bar{b}_{x_{m}}$ where $m \leqq$ $n / 2$. Every member $b$ of $C_{n}$ belongs to a unique $C(K, n)$ (namely, if $b=b_{x_{1}} \wedge \cdots \wedge b_{x_{k}} \wedge \bar{b}_{x_{k+1}} \wedge \cdots \wedge \bar{b}_{x_{m}}$ then $b \in C(K, n)$ if and only if $K$ is the set of all $\langle i, j\rangle$, such that $2 \leqq i \leqq n, i \leqq j<i^{2}$, and for some $\left.r x_{r} \in T_{i, j}\right)$. Hence $\left\{C(K, n) \mid K \cong K_{n}\right\}$ constitutes a partition of $C_{n}$.

The power of $K_{n}$ is $\sum_{i=2}^{n} i^{2}$, hence there are $2^{\Sigma_{i=1}^{n} i^{2}}$ different subsets of $K_{n}$. Consequently every subset of $C_{n}$, in which the meet of every 2 different elements is in $\Im$ and is of power $\leqq p_{n}$, where $p_{n}=2^{(n / 2)+\Sigma_{i=1}^{n} i^{2}}$. Put $C_{n}^{\prime}=\left\{b|b \in| \mathfrak{B} \mid\right.$ and $b \geqq b^{\prime}$ for some $b^{\prime}$ in $\left.C_{n}\right\}$. It is clear 
that every subset of $C_{n}^{\prime}$ in which the meet of every 2 different elements is in $\mathfrak{\Im}$ is of power $\leqq p_{n}$.

Every element of $\mathfrak{B}$ is a join of meets of free generators and complements of free generators, if all the meets are in $\Im$ the element is in $\Im$. Consequently if $b \notin \Im$ then $b \geqq b^{\prime}$ for some $b^{\prime}$, not belonging to $\Im$, which is a meet of free generators ond complements of free generators. Hence $\bigcup_{n=2}^{\infty} C_{n}^{\prime}$ is the set of all elements of $\mathfrak{B}$ which are not in $\Im$.

Put $D_{n}=\left\{b / \mathfrak{\Im} \mid b \in C_{n}^{\prime}\right\} \cup\{\mathfrak{O} / \mathfrak{F}\}$. Then $|\mathfrak{B} / \mathfrak{F}|=\bigcup_{n=2}^{\infty} D_{n}$ and in $D_{n}$ there are at most $p_{n}+1$ disjoint elements. Consequently (II) follows from Lemma 1.2.

(III) There is no strictly positive measure on $\mathfrak{B} / \Im$.

Proof. By contradiction. If there is a strictly positive measure on $\mathfrak{B} / \mathfrak{S}$ then there are sets $B_{1}, \cdots, B_{n}, \cdots, 1 \leqq n<\infty$, such that $|\mathfrak{B} / \mathfrak{\Im}|=\{\mathrm{O} / \mathfrak{\Im}\} \cup \mathrm{U}_{n=1}^{\infty} B_{n}$ and, for all $n$, Int $\left(B_{n}\right)>0$. As we have seen $b_{x_{1}} \wedge \cdots \wedge b_{x_{k}} \in \mathfrak{\Im}$ if and only if for some $i$ we have $k \geqq i \geqq 2$ and among $x_{1}, \cdots, x_{k}$ there are $i$ points in $i$ different intervals of the form $T_{i, j}$. In particular $b_{x} \notin \Im$ for all $x \in(0,1)$. Hence if we put $X_{n}=\left\{x \mid b_{x} \in B_{n}\right\}$ we get $\bigcup_{n=1}^{\infty} X_{n}=(0,1)$. Consequently some $X_{n}$ must be dense in some open non empty interval. Let $X_{k}$ be dense in the interval $T,(T \neq \phi)$.

Among $T_{2}, T_{3}, \cdots, T_{n}, \cdots$ there are infinitely many subintervals of $T$, let them be $T_{n(1)}, \cdots, T_{n(j)}, \cdots$ where $n(i)<n(j)$ if $i<j$. Consider $T_{n(i), 1}, \cdots, T_{n(i), n(i)^{2}}$, there are points $x_{1}, \cdots, x_{n(i)^{2}}$ in $X_{k}$ such that $x_{j} \in T_{n(i), j}$ for all $1 \leqq j \leqq n(i)^{2}$. Consider the $n(i)^{2}$-tuple $\left\langle b_{x_{1}} / \mathfrak{F}, \cdots, b_{x_{j}} / \mathfrak{F}, \cdots\right\rangle_{j \leqq n(i)^{2}}$. This is a tuple of members of $B_{k}$. Since by our definition every meet of $n(i)$ elements among $b_{x_{1}}, \cdots, b_{x_{n(i)}{ }^{2}}$ is in $\Im$ we get $N\left(\left\langle b_{x_{1}} / \mathfrak{\Im}, \cdots\right\rangle\right) \leqq n(i)$, hence $\left(1 / n(i)^{2}\right) \cdot N\left(\left\langle b_{x_{1}}, \cdots\right\rangle\right) \leqq(1 / n(i))$. Consequently $\operatorname{Int}\left(B_{k}\right) \leqq(1 / n(i))$; letting $i \rightarrow \infty$ we get $\operatorname{Int}\left(B_{k}\right)=0$. Contradiction.

Corollary. The C.C.C. is not sufficient for the existence of a strictly positive measure.

THEOREM 2.2. There is a Boolean algebra $\mathfrak{2}$ satisfying (*) and having the property:

$(\dagger)$ If $a$ is any nonzero member of $\mathfrak{U}$ then $\left.\mathfrak{U}\right|_{a}$ has no strictly positive measure.

Theorem 2.2 follows from Theorem 2.1, since, as it is not difficult to show, if $\mathfrak{A}$ has no strictly positive measure and satisfies the C.C.C. then, for some nonzero element $b,\left.\mathfrak{A}\right|_{b}$ satisfies ( $\dagger$ ). Obviously if $\mathfrak{A}$ satisfies $(*)$ so does $\left.\mathfrak{A}\right|_{b}$. Another way (suggested to the author by 
R. S. Pierce) is, given $\mathfrak{X}$, to construct the free product, $\mathfrak{A}^{\prime}$, of $\boldsymbol{\aleph}_{0}$ copies of $\mathfrak{2}$. (This corresponds to taking the cartesian product of $\aleph_{0}$ copies of the Stone representation of. $\mathfrak{A}$ with the product topology). It can be shown then that if $\mathfrak{A}$ has no strictly positive measure then $\mathfrak{A}^{\prime}$ satisfies $(\dagger)$ and if $\mathfrak{A}$ satisfies $(*)$ so does $\mathfrak{\mathfrak { X } ^ { \prime }}$.

Note that $(\dagger)$ is equivalent to:

( $\ddagger$ Whenever $m$ is a measure on $\mathfrak{A}$ then $\{b \mid m(b)=0\}$ is dense in $\mathfrak{A}$. (If $\mathfrak{A}$ satisfies $(\dagger), m$ is a measure on $\mathfrak{A}$, and $m(a)>0$ then define $m^{\prime}$ on $\left.\mathfrak{A}\right|_{a}$ by: $m^{\prime}(b)=(1 / m(a)) \cdot m(b)$ for all $b \leqq a$. Because of $(\dagger)$ there is an element $b$ such that $a \geqq b>0$ and $m^{\prime}(b)=0$, for this $b$ we have $m(b)=0$. The implication $(\ddagger) \rightarrow(\dagger)$ is as easy.)

Because of Theorem 2.2 it is easily seen that both Theorem 2.1 and Theorem 2.2 hold if we replace "strictly positive measure" by "strictly positive measure"" where by a measure' we understand a finitely additive function into $[0, \infty]$ which is finite for some nonzero element. Property $(t)$ is equivalent to the corresponding property ( + '), obtained by replacing "measure" by "measure’." ( $\ddagger$ ) is equivalent to the property that whenever $m$ is a measure' on $\mathfrak{A}$ then $\{b \mid m(b)=0\}$ is dense in the set of all elements of finite measure. It should be noted, however, that $(*)$ is not a necessary condition for the existence of a measure'.

Section 3. The problems concerning the conditions for the existence of strictly positive measures arise also for countably additive Boolean algebras (i.e., where all countable joins and meets exist) and countably additive measures (i.e., where $m\left(\mathbf{V}_{i=1}^{\infty} a_{i}\right)=\sum_{i=1}^{\infty} m\left(a_{i}\right)$, whenever $a_{i} \wedge a_{j}=0$ for all $i \neq j$ ). A characterization of the countably additive Boolean algebras which have a strictly positive countably additive measure was given by Maharam [6]. If we take the completion by cuts of the Boolean algebra whose existence is claimed in: Theorem 2.1 we get a complete, hence countably additive, Boolean algebra which satisfies $(*)$, and which has no strictly positive measure, hence, a fortiori, no strictly positive countably additive measure. However it turns out that the solution of this problem for the countably additive case is much easier. The completion by cuts of the free Boolean algebra on $\boldsymbol{\aleph}_{0}$ generators is also a Boolean algebra. which satisfies $(*)$ and has no strictly positive countably additive measure (although it does have a strictly positive finitely additive: measure). It turns out that the interesting problem for the countably additive case involves an additional condition, the so-called weak countable distributivity.

A countably additive Boolean algebra is countably distributive if for every double sequence of elements $a_{m, n}, 0<m<\infty$ and $0<n<\infty$, the join $\mathbf{V}_{k} \mathbf{V}_{m=1}^{\infty} a_{m, k_{m}}$, where $k$ ranges over all sequences of natural 
numbers, exists and is equal to $\bigwedge_{m=1}^{\infty} \bigvee_{n=1}^{\infty} a_{m, n}$. It is weakly countably distributive if this holds for all double sequences $a_{m, n}$ in which $a_{m, 1} \leqq$ $a_{m, 2} \leqq \cdots \leqq a_{m, n} \leqq a_{m, n+1} \leqq \cdots$ for all $m$. As was pointed out by Maharam [6, pp. $158 \mathrm{ff}$, weak countable distributivity is necessary for the existence of strictly positive countably additive measures. The analogous problems for the countably additive case are whether either condition $(*)$ or the C.C.C. together with weak countable distributivity is sufficient for the existence of a strictly positive countably additive measure. No answer to either of these questions is known. The completion by cuts of the Boolean algebra used in the proof of Theorem 2.1 is, unfortunately, not weakly countably distributive.

Section 4. Connections with Souslin's conjecture. Souslin's conjecture (Fund. Math. 1, 1920, p. 223) is that every non empty ordered set without jumps (i.e. between every two elements there is another element) and without gaps (i.e. every bounded subset has a least upper bound) which has neither a first nor a last element and which has at most countably many pairwise disjoint intervals, is order isomorphic to the real line.

As was shown by Horn and Tarski [3, pp. 487 f.], if the C.C.C. implies $(*)$ then Souslin's conjecture is true. Thus, a fortiori, the sufficiency of the C.C.C. for the existence of a strictly positive measure would imply Souslin's conjecture, hence the corollary to Theorem 1 could be expected. The problem to show that the C.C.C. does not imply (*) was stated in [3] and remains open. Also as was proved by Maharam [6, pp. $164 \mathrm{ff}$.$] , the sufficienty of the C.C.C. together with$ weak countably distributivity for the existence of strictly positive countably additive measures implies Souslin's conjecture, hence it is unlikely to be proved.

We will show that Souslin's conjecture is equivalent to some relatively simple statements concerning countably distributive Boolean algebras. To do this we use a well known formulation of Souslin's conjecture in terms of trees, originally due to Kurepa [5, pp. $122 \mathrm{ff}$.].

By a tree we mean a system $\langle T, \geqq\rangle$ where $\geqq$ is a partial ordering of $T$ such that for all $a \in T$ the set $\{b \mid b \geqq a\}$ is well ordered by $\geqq$. The rank of $a$ is the ordinal which is order isomorphic to $\{b \mid b \geqq a\}$. The tree can be best visualized by "placing" its elements on different "levels" according to their rank. Two elements, $a$ and $b$, are incomparable if neither $a \geqq b$ nor $b \geqq a$. A path is a maximal subset of $T$ which is totally ordered by $\geqq$. Every path is, of course, well ordered by $\geqq$ and the ordinal which is order isomorphic to it is the length of the path. (Of course every ordered subset of $T$ can be extended to a path.) Let $\omega_{1}$ be the first uncountable ordinal. Souslin's conjecture is equivalent to the following statement: 
Every uncountable tree has either a path of length $\geqq \omega_{1}$ or has $\aleph_{1}$ pairwise incomparable elements.

The proof of the following lemma is essentially due to Maharam $[6, p .166]$.

LEMMA 4.1. Let $\langle T, \geqq\rangle$ be a tree which has no $\aleph_{1}$ pairwise incomparable elements and in which $\{b \mid b \geqq a\}$ is countable for every $a \in T$. Let $m$ be a function from $T$ into $[0, \infty]$ such that $a \geqq b \Rightarrow$ $m(a) \geqq m(b)$, and if $m(a)>0$ then, for some $b, a>b$ and $m(a)>m(b)$. Then $\{a \mid m(a)>0\}$ is countable.

To prove it put $S_{r}=\{a \mid m(a) \leqq r$ and for every $b$ if $b>a$ then $m(b)>r\}, \quad(r$ is any real number). It follows that if $m(a) \leqq r$ then $a^{\prime} \geqq a$ for some $a^{\prime}$ in $S_{r}$. Also $S_{r}$ is a set of incomparable elements and hence countable. If $m(a)>0$ then $m(a)>m(b)$ for some $b$ such that $a>b$. If $r$ is a rational number between $m(a)$ and $m(b)$ then $b^{\prime} \geqq b$ for some $b^{\prime} \in S_{r}$ and it follows that $a>b^{\prime}$. Hence $\{a \mid m(a)>0\}=$ $\left\{a \mid a>b\right.$ for some $b \in S_{r}$ and some positive rational $\left.r\right\}$. Since for every $b\{a \mid a>b\}$ is countable, $\{a \mid m(a)>0\}$ is countable.

An atom of a Boolean algebra is a nonzero element, $a$, such that whenever $b \leqq a$ either $b=0$ or $b=a$.

The following theorem establishes the equivalence of Souslin's conjecture and several statements concerning Boolean algebras.

THEOREM 4.1. ${ }^{1}$ Souslin's conjecture is equivalent to each one of the following statements

(i) Every countably additive and countably distributive Boolean algebra satisfying the C.C.C. is isomorphic to the Boolean algebra of all subsets of a countable set.

(ii) Every countably additive and countably distributive Boolean

1 The ideas used in proving this theorem are known and similar results have been proved. As was pointed out to the author, the implication 'Souslin's conjecture $\Rightarrow$ (i)' can be derived using some results of Horn and Tarski [3, p. 480, Th. 2.3 and p. 487, Th. 2.11], and the equivalence "Souslin's conjecture $\Leftrightarrow(i)$ " was proved by Maharam, $[7$, p. 590]. The construction which is used here to show that (v) implies Souslin's conjecture is essentially the same as that used by Maharam [6, pp. 164 ff.] to prove a weaker implication, the main difference being that "weakly countably distributive" is used there instead of "countably distributive." This construction was found and used independently by the author to prove that (iv) implies Souslin's conjecture, the main argument by which the measure in (iv) can be replaced by a more general function is due to Maharam [6, p. 166]. Using the original formulation of Souslin's conjecture, which involves ordered sets instead of trees, Kelley [4, p. 1172] proved an implication weaker than '(iv) $\Longrightarrow$ Souslin's conjecture,' the difference being, again, the use of "weakly countably distributive" instead of "countably distributive." For other results concerning the connections between Souslin's conjecture and Boolean algebras see [4], [7], and [3] where more references are given. 
algebra satisfying the C.C.C. has a strictly positive countably additive measure.

(iii) Every countable additive and countably distributive Boolean algebra satisfying the C.C.C. has a strictly positive measure.

(iv) Every countably additive and countably distributive Boolean algebra satisfying the C.C.C. has a measure $m$ such that the maximal element, 1, is not a countable join of elements of measure 0.

(v) Like (iv) except that $m$ is required to be only a monotonic function having nonnegative values, such that whenever $m(a)>0$ and $a$ is not an atom then $m(a)>m(b)$ for some nonzero $b$ which is $<a$.

Since every countably additive Boolean algebra satisfying the C.C.C. is complete, we can replace in (i)-(v) "countably additive and countably distributive" by "complete and countably distributive."

The following is a sketch of the proof. First to show that Souslin's conjecture implies (i), assume that $\mathfrak{B}$ is a countably additive, countably distributive Boolean algebra satisfying the C.C.C. By the axiom of choice it follows that there are two functions $f_{0}$ and $f_{1}$ defined for all nonzero elements $a$ which are not atoms, such that $f_{0}(a) \vee f_{1}(a)=a$, $f_{0}(a) \wedge f_{1}(a)=0$, and $f_{0}(a), f_{1}(a) \neq 0$. We now construct a set of sequences, $S$, of 0 's and 1 's, whose lengths are countable ordinals, and a mapping $s \rightarrow a_{s}$, defined for all $s \in S$, such that $a_{s} \in|\mathfrak{B}|$. This is done as follows:

(1) $\langle 0\rangle \in S$ and $a_{0}=1$

(2) If $s=\left\langle s_{0}, \cdots, s_{\lambda}, \cdots\right\rangle_{\lambda<\infty}$ is in $S$ and if $a_{s}$ is not an atom then $s 0$ and $s 1$, defined respectively as $\left\langle s_{0}, \cdots, s_{\lambda}, \cdots, 0\right\rangle$ and $\left\langle s_{0}, \cdots, s_{\lambda}, \cdots, 1\right\rangle$, are in $S, a_{s 0}=f_{0}\left(a_{s}\right)$ and $a_{s 1}=f_{1}\left(a_{s}\right)$. If $a_{s}$ in an atom then $s 0 \in S$ and $a_{s}=a_{s 0}$.

(3) Let $\left\{s^{i} \mid i<\omega_{0}\right\}$ be a subset of $S\left(\omega_{0}=\right.$ the first infinite ordinal). Let $\alpha_{i}$ be the length of $s^{i}$. If $\alpha_{0}<\alpha_{1}<\cdots \alpha_{i}<\alpha_{i+1} \cdots$ and $s_{\lambda}^{i}=s_{\lambda}^{j}$ for all $\lambda<\alpha_{i}, \alpha_{j}$, and if $\bigwedge_{i<\omega_{0}} a_{s i} \neq 0$, then the sequence $s$, whose length is $\bigcup_{i<\omega_{0}} \alpha_{i}$, such that $s_{\lambda}=s_{\lambda}^{i}$ for all $\lambda<\alpha_{i}\left(i<\omega_{0}\right)$, is in $S$, and $a_{s}=\bigwedge_{i<\omega_{0}} a_{s i}$.

(4) $S$ contains no other sequences.

Let $S_{a}$ be the set of all members of $S$ of length $\alpha$. From the countable distributivity of $\mathfrak{B}$ it follows that $1=\mathbf{V}_{s \in s_{\alpha}} a_{s}$ whenever $\alpha$ is countable. $\left\{a_{s} \mid s \in S\right\}$ together with the partial ordering of $\mathfrak{B}$ form a tree. In this tree $a_{s} \leqq a_{s^{\prime}}$ or $a_{s^{\prime}} \leqq a_{s}$ or $a_{s} \wedge a_{s^{\prime}}=0$. By the C.C.C. this tree has no path of length $>\omega_{1}$, and no $\aleph_{1}$ pairwise incomparable elements. By Souslin's conjecture the tree is countable. Hence for some countable $\alpha$ every $a_{s}$, where $s \in S_{\alpha}$, is an atom. From this it easily follows that $\mathfrak{B}$ is isomorphic to the Boolean algebra of all subsets of the set of all its atoms. The set of all its atoms is, of course, countable. 
Each of the conditions (i)-(iv) is easily seen to imply its successor. It remains to show that (v) implies Souslin's conjecture. Assume Souslin's conjecture is false and let $\langle T, \geqq\rangle$ be an uncountable tree with no path of length $\omega_{1}$ and no $\boldsymbol{\aleph}_{1}$ pairwise incomparable elements. For every $a$ in $T$ let $P(a)$ be the set of all paths containing $a$, let $P$ be the set of all paths and for every ordinal $\alpha$ let $P_{a}$ be the set of all paths of length $\leqq \alpha$. It follows easily that $P \neq P_{a}$ whenever $\alpha$ is countable. Let $\mathfrak{B}$ be the countably additive Boolean algebra of subsest of $P$ generated by $\{P(a) \mid a \in T\}$. Let $\Im=\left\{A \mid A \subseteq P_{\alpha}\right.$ for some countable $\alpha\}$. $\Im$ is a countably additive ideal of $\mathfrak{B}$ and $\mathfrak{B} / \mathfrak{\Im}$ is a countably additive, countably distributive Boolean algebra satisfying the C.C.C.

A proof of this will proceed along the following steps:

If $a \geqq b$ then $P(a) \supseteqq P(b)$ and if $a$ and $b$ are incomparable then $P(a) \cap P(b)=\phi$. Consequently if $P(a) \cap P(b) \neq \phi$ then $P(a) \supseteqq P(b)$ or $P(b) \supseteqq P(a)$. If $A=U_{i \in I} P\left(a_{i}\right)$ then for every $j \in I$ the set of all $a_{i}$ 's, $i \in I$, such that $a_{i} \geqq a_{j}$, is well ordered by $\geqq$ and has a first element $a_{i j}$; obviously $A=U_{j \in I} P\left(a_{i_{j}}\right)$ and $\left\{a_{i}, j \in I\right\}$ is a set of pairwise incomparable elements. Thus every union of $P(a)$ 's is also a union of $P(a)$ 's which are pairwise disjoint. Now consider all sets of the form: (\#) $A \cup A^{\prime}$, where $A$ is a countable union of pairwise disjoint $P(a)$ 's and $A^{\prime} \subseteq P_{\alpha}$ for some countable $\alpha$.

The complement of any $P(a)$ is of this form, namely if $a$ is of rank $\alpha$ then complement of $P(a)=A \cup A^{\prime}$ where $A$ is the union of all $P(b)$ 's such that $b$ is of rank $\alpha$ and $b \neq a$, and $A^{\prime}$ is the subset of $P_{\alpha}$ consisting of all paths not containing $a$. A countable union of sets of the form (\#) is obviously of this form and this, although less obvious, is also true for countable intersections. (If $B_{n}=$ $U_{i \in I_{n}} P\left(a_{i}\right) \cup A_{n}^{\prime}$, where $I_{n}$ is countable and $A_{n}^{\prime} \subseteq P_{a_{n}}, n=0,1, \cdots$, then let $\alpha$ be any countable ordinal bigger than the ranks of all $a_{i}$ 's, $i \in I_{n}, n=0,1, \cdots$, and all the $\alpha_{n}$ 's. Taking the intersection of the $B_{n}$ 's and distributing one gets a set of paths each of length $\leqq \alpha$ as well as sets of the form $\bigcap_{n=0}^{\infty} P\left(a_{i_{n}}\right), i_{n} \in I_{n}$. It is easily seen that every set of the last form is either empty or consists of a single path of length $\leqq \alpha$ or is a countable union of $P(a)$ 's where the $\alpha$ 's are all of rank $\leqq \alpha$. Since there are countable many $a$ 's of rank $\leqq \alpha$ the assertion follows.) Hence all members of $\mathfrak{B}$ are of form (\#). Consequently every member of $\mathfrak{B} / \mathfrak{\Im}$ is of the form $U_{i \in I} P\left(a_{i}\right) / \mathfrak{\Im}$, where $I$ is countable, $P\left(a_{i}\right) \notin \Im$ for all $i \in I$ and $P\left(a_{i}\right) \cap P\left(a_{j}\right)=\phi$ if $\mathrm{i} \neq j$; the member is 0 if and only if $I=\phi$. If $x_{m, n}=U_{i \in I_{m, n}} P\left(a_{i}\right) / \mathfrak{\Im}$, and $\Lambda_{m=0} \bigvee_{n=0} x_{m, n} \neq 0$ then, for some $a$ such that $P(a) \notin \Im$ and rank $a \geqq \operatorname{rank} a_{i}$ for all $i \in \bigcup_{m=0}^{\infty} \bigcup_{n=0}^{\infty} I_{m, n}, \bigwedge_{n=0}^{\infty} x_{m, n} \geqq P(a) / \Im$ for all $m$. From the properties of the $P(a)$ 's it follows that for every $m$ there is an $n_{m}$ such that $x_{m, n_{m}} \geqq P(a) / \Im$, hence $\Lambda_{m=0}^{\infty} x_{m, n_{m}} \neq 0$. This is well known to imply 
the countable distributivity of $\mathfrak{B} / \mathfrak{\Im}$.

From the properties of the $P(a)$ 's it follows that $\{P(a) / \Im \mid a \notin \Im\}$ together with the partial ordering of $\mathfrak{B} / \mathfrak{\Im}$, restricted to this set, form a tree having the properties required in Lemma 4.1. If $m$ is a function on $|\mathfrak{B} / \mathfrak{\Im}|$ satisfying the conditions mentioned in $(V)$ then its restriction to $\{P(a) / \Im \mid a \notin \Im\}$ will have the properties required in Lemma 4.1 (this is so because no $P(a) / \mathfrak{S}$ is an atom in $\mathfrak{B} / \mathfrak{\Im}$, and, as was mentioned before, every member of $\mathfrak{B} / \mathfrak{F}$ is a join of $P(a)$ 's). By Lemma 4.1, $\{a \mid m(P(a) / \Im)>0\}$ is countable. This implies that, for some countable $\alpha, m(P(\alpha) / \Im)=0$ for all $a$ 's of rank $\alpha$; but for every countable $\alpha\{P(a) / \mathfrak{\Im} \mid \alpha$ is of rank $\alpha\}$ is countable and its join is 1 .

\section{REFERENCES}

1. G. Birkhoff, Lattice theory, Amer. Math. Soc. Colloquium Publications, Vol. XXX, Providence, 1948.

2. - On the structure of abstract algebras, Proc. Camb. Phil. Soc. Vol., 31 (1935), 433-454.

3. A. Horn and A. Tarski, Measures in Boolean algebras, Trans. Amer. Math. Soc., 64 (1948), 467-497.

4. J. L. Kelley, Measures in Boolean algebras, Pacific J. Math., 9 (1165-1177).

5. G. Kurepa, Ensemble ordounes et ramifies, theses, Publication Mathematiques de l'Universite de Belgrade, 4 (1935), 1-138.

6. D. Maharam, An algebraic characterization of measure algebras, Ann. of Math., 48 (1947), 154-167.

7. - Set functions and Souslin's hypothesis, Bull. Amer. Math. Soc., 54 (1948), 588-590.

8. A. Tarski, Ideale in vollständigen Mengenkorpern, I, Fund. Math., 33 (1939), 45-63.

9. _ Ideale in vollständigen Mengenkorpern, II, Fund. Math., 33 (1945), 51-63.

(Date of reprint 1939)

UNIVERSITY OF COLUMBIA 



\section{PACIFIC JOURNAL OF MATHEMATICS}

\section{EDITORS}

Robert Osserman

Stanford University

Stanford, California

M. G. Arsove

University of Washington

Seattle 5 , Washington
J. DugundjI

University of Southern Califorma: Los Angeles 7, California

Lowell J. Paige

University of California

Los Angeles 24, California

\section{ASSOCIATE EDITORS}

E. F. BECKENBACH

B. H. NeumanN

F. WOLF

K. YosIDA

\section{SUPPORTING INSTITUTIONS}

UNIVERSITY OF BRITISH COLUMBIA

CALIFORNIA INSTITUTE OF TECHNOLOGY

UNIVERSITY OF CALIFORNIA

MONTANA STATE UNIVERSITY

UNIVERSITY OF NEVADA

NEW MEXICO STATE UNIVERSITY

OREGON STATE UNIVERSITY

UNIVERSITY OF OREGON

OSAKA UNIVERSITY

UNIVERSITY OF SOUTHERN CALIFORNIA
STANFORD UNIVERSITY

UNIVERSITY OF TOKYO

UNIVERSITY OF UTAH

WASHINGTON STATE UNIVERSITY

UNIVERSITY OF WASHINGTON

AMERICAN MATHEMATICAL SOCIETY CALIFORNIA RESEARCH CORPORATION SPACE TECHNOLOGY LABORATORIES NAVAL ORDNANCE TEST STATION 


\section{Pacific Journal of Mathematics}

\section{Vol. 14, No. 1 \\ May, 1964}

Richard Arens, Normal form for a Pfaffian .........................

Charles Vernon Coffman, Non-linear differential equations on cones in Banach

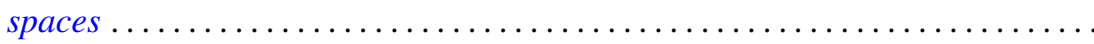

Ralph DeMarr, Order convergence in linear topological spaces ..............

Peter Larkin Duren, On the spectrum of a Toeplitz operator ................

Robert E. Edwards, Endomorphisms of function-spaces which leave stable all

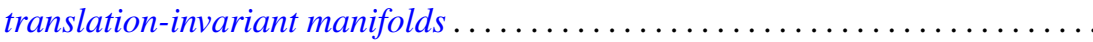

Erik Maurice Ellentuck, Infinite products of isols . . . . . . . . . . . . . . . . 49

William James Firey, Some applications of means of convex bodies . . . . . . . . 53

Haim Gaifman, Concerning measures on Boolean algebras ............. 61

Richard Carl Gilbert, Extremal spectral functions of a symmetric operator. . . . . . 75

Ronald Lewis Graham, On finite sums of reciprocals of distinct nth powers ..... 85

Hwa Suk Hahn, On the relative growth of differences of partition functions ...... 93

Isidore Isaac Hirschman, Jr., Extreme eigen values of Toeplitz forms associated

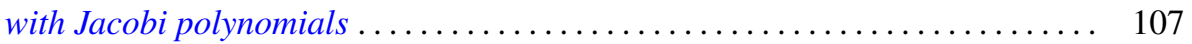

Chen-jung Hsu, Remarks on certain almost product spaces . . . . . . . . . . . 163

George Seth Innis, Jr., Some reproducing kernels for the unit disk . . . . . . . . . 177

Ronald Jacobowitz, Multiplicativity of the local Hilbert symbol . . . . . . . . . . . 187

Paul Joseph Kelly, On some mappings related to graphs ................. 191

William A. Kirk, On curvature of a metric space at a point . . . . . . . . . . . . 195

G. J. Kurowski, On the convergence of semi-discrete analytic functions . . . . . . . 199

Richard George Laatsch, Extensions of subadditive functions . . . . . . . . . . . 209

V. Marić, On some properties of solutions of $\Delta \psi+A\left(r^{2}\right) X \nabla \psi+C\left(r^{2}\right) \psi=0 \ldots 217$

William H. Mills, Polynomials with minimal value sets . . . . . . . . . . . 225

George James Minty, Jr., On the monotonicity of the gradient of a convex

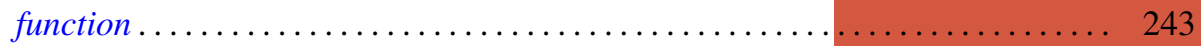

George James Minty, Jr., On the solvability of nonlinear functional equations of 'monotonic' type ................................... 249

J. B. Muskat, On the solvability of $x^{e} \equiv e(\bmod p) \ldots \ldots \ldots \ldots \ldots \ldots \ldots \ldots . \ldots \ldots$

Zeev Nehari, On an inequality of $P . R$. Bessack ................... 261

Raymond Moos Redheffer and Ernst Gabor Straus, Degenerate elliptic

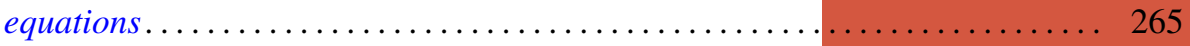

Abraham Robinson, On generalized limits and linear functionals . . . . . . . . . 269

Bernard W. Roos, On a class of singular second order differential equations with a

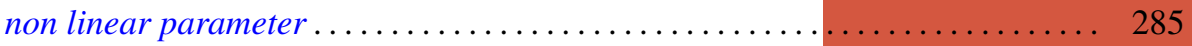

Tôru Saitô, Ordered completely regular semigroups . . . . . . . . . . . . . . . . 295

Edward Silverman, A problem of least area ....................... 309

Robert C. Sine, Spectral decomposition of a class of operators . . . . . . . . . 333

Jonathan Dean Swift, Chains and graphs of Ostrom planes . . . . . . . . . . . 353

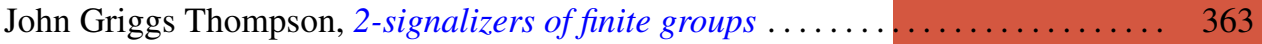

Harold Widom, On the spectrum of a Toeplitz operator . . . . . . . . . . . . . 365 